PROCEEDINGS OF THE

AMERICAN MATHEMATICAL SOCIETY

Volume 140, Number 4, April 2012, Pages 1349-1352

S 0002-9939(2011)11043-8

Article electronically published on August 4, 2011

\title{
ON THE NORMS OF DISCRETE ANALOGUES OF CONVOLUTION OPERATORS
}

\author{
OLEG KOVRIZHKIN
}

(Communicated by Michael T. Lacey)

\begin{abstract}
We consider a discrete analogue of convolution operator $T(f)=$ $K * f$ from $L^{p}\left(\mathbb{R}^{d}\right)$ to $L^{p}\left(\mathbb{R}^{d}\right): T_{\text {dis }}(g)=K_{\text {dis }} * g$ from $\ell^{p}\left(\mathbb{Z}^{d}\right)$ to $\ell^{p}\left(\mathbb{Z}^{d}\right)$ where $K_{\text {dis }}=\left.K\right|_{\mathbb{Z}^{d}}$ and $\hat{K}$ is supported in the fundamental cube. We show that the estimate $\left\|T_{d i s}\right\|_{p} \leq C^{d}\|T\|_{p}$ with $C>1$ cannot be improved for a certain range of $p$.
\end{abstract}

We consider a convolution operator $T(f)=K * f$ from $L^{p}\left(\mathbb{R}^{d}\right)$ to $L^{p}\left(\mathbb{R}^{d}\right)$ with a distribution kernel $K$ whose Fourier transform $\hat{K}(\xi)$ is supported in the fundamental cube $Q=\left\{\xi=\left(\xi_{1}, \xi_{2}, \ldots, \xi_{d}\right):-\frac{1}{2}<\xi_{i} \leq \frac{1}{2}, i=1,2, \ldots, d\right\}=\left(-\frac{1}{2}, \frac{1}{2}\right]^{d}$. The Fourier transform $(T(f))^{\wedge}=\hat{K}(\xi) \hat{f}(\xi)$. It is well known that if $T(f)=K * f$ acts from $L^{p}$ to $L^{p}$, then it also acts from $L^{p^{\prime}}$ to $L^{p^{\prime}}$ with the same norm. Thus we restrict ourselves to the case $1 \leq p \leq 2$. In particular, we can say that $T(f)=K * f$ is an operator from $L^{r}$ to $L^{r}$ where $p \leq r \leq 2$. We will be interested in the norms of $T$ from $L^{p}$ to $L^{p}$. If $p=1$, then $\|T\|_{1}=\|K\|_{1}$, and if $p=2$, then $\|T\|_{2}=\|\hat{K}\|_{\infty}$. Interpolating, we get $\|T\|_{p} \leq\|K\|_{1}^{\frac{2}{p}-1}\|\hat{K}\|_{\infty}^{2-\frac{2}{p}}$ for $1<p<2$.

As we mentioned before, $T$ is also a bounded operator from $L^{2}$ to $L^{2}$ and therefore $\hat{K}(\xi)$ is bounded, which makes $K(x)=\int_{\mathbb{R}^{d}} \hat{K}(\xi) e^{i 2 \pi \xi x} d \xi$ an infinitely differentiable function extendable to an entire function of exponential type. We define $K_{d i s}=$ $\left.K\right|_{\mathbb{Z}^{d}}$ and $T_{d i s}(g)=K_{d i s} * g$ as an operator on $\mathbb{Z}^{d}$ :

$$
T_{d i s}(g)(n)=\sum_{m \in \mathbb{Z}^{d}} K(n-m) g(m) .
$$

Since $\hat{K}$ is supported in the fundamental cube $Q$, we can view $K(n)$ as its Fourier coefficients and thus $\hat{K}(\xi)=\sum_{n \in \mathbb{Z}^{d}} K(n) e^{i 2 \pi n \xi} \chi_{Q}(\xi)$; i.e., $K_{\text {dis }}$ and $K$ are determined by each other. We will be interested in norms of $T_{d i s}$ from $\ell^{p}\left(\mathbb{Z}^{d}\right)$ to $\ell^{p}\left(\mathbb{Z}^{d}\right)$. What was said above about $\|T\|_{p}$ is also true for $\left\|T_{d i s}\right\|_{p}$. If $p=1$, then $\left\|T_{d i s}\right\|_{1}=$ $\left\|K_{d i s}\right\|_{1}$, and if $p=2$, then $\left\|T_{d i s}\right\|_{2}=\|\hat{K}\|_{\infty}$. Interpolating, we get $\left\|T_{d i s}\right\|_{p} \leq$ $\left\|K_{d i s}\right\|_{1}^{\frac{2}{p}-1}\|\hat{K}\|_{\infty}^{2-\frac{2}{p}}$ for $1<p<2$. We will find the following estimate useful.

Received by the editors December 16, 2010 and, in revised form, January 4, 2011.

2010 Mathematics Subject Classification. Primary 42A99, 42B99.

Key words and phrases. Discrete analogues, convolutions.

This research was partially supported by NSF grant DMS 0201099 . 


\section{Proposition 1.}

$$
\left\|T_{d i s}\right\|_{p} \geq\left\|K_{d i s}\right\|_{p} .
$$

To show this, just take $g(n)=\delta_{0}(n)$.

The main question we are interested in is how $\left\|T_{d i s}\right\|_{p}$ is controlled by $\|T\|_{p}$. As we said earlier, we restrict ourselves to the case $1 \leq p \leq 2$ since $\left\|T_{d i s}\right\|_{p^{\prime}}=\left\|T_{d i s}\right\|_{p}$ and $\|T\|_{p^{\prime}}=\|T\|_{p}$. The following result is due to Magyar, Stein and Wainger 1, Proposition 2.1]: If $T$ is a bounded operator from $L^{p}\left(\mathbb{R}^{d}\right)$ to $L^{p}\left(\mathbb{R}^{d}\right)$, then $T_{\text {dis }}$ is a bounded operator from $\ell^{p}\left(\mathbb{Z}^{d}\right)$ to $\ell^{p}\left(\mathbb{Z}^{d}\right)$ and

$$
\left\|T_{\text {dis }}\right\|_{p} \leq C(d)\|T\|_{p}
$$

where $C$ depends only on the dimension d but not on $p$.

The actual statement is more general. Magyar, Stein and Wainger raised the following question in their paper (see Remark 1 after Proposition 2.1 in [1]): Is it possible to take $C$ independently of the dimension $d$ and, in particular, if $C=1$ ?

We will answer this question negatively.

Theorem 1. There is $p_{0}$ with $1<p_{0}<2$ such that for all $p$ with $1 \leq p<p_{0}$ the optimal $C(d)$ in (2) has a lower bound $C(d) \geq B^{d}$ where $B>1$.

Actually the proof of Proposition 2.1 in 1 shows that $C(d) \leq A^{d}$, and thus this estimate of $C(d)$ cannot be improved. If $p=2$, then $\|T\|_{2}=\left\|T_{\text {dis }}\right\|_{2}=\|\hat{K}\|_{\infty}$. Therefore, $C(d)=1$ for $p=2$. If $K \geq 0$, then $\|T\|_{p}=\left\|T_{\text {dis }}\right\|_{p}=\|\hat{K}\|_{\infty}=\|K\|_{1}$. Thus $C(d) \geq 1$.

Proof of Theorem 1. First we consider the case when the dimension $d=1$ and $p=$ 1. We will construct a kernel $K$ such that $\left\|T_{\text {dis }}\right\|_{1}=\sum_{n \in \mathbb{Z}}|K(n)|>\int_{\mathbb{R}}|K(x)| d x=$ $\|T\|_{1}$. Let $\phi(x)$ be a nonnegative Schwartz function whose Fourier transform $\hat{\phi}(\xi)$ is also nonnegative and supported in $\left[-\frac{1}{2}, \frac{1}{2}\right]$, for example, $\phi(x)=\psi(x) \cdot \bar{\psi}(x)$ where $\hat{\psi}(\xi)$ is nonnegative and supported in $\left[-\frac{1}{4}, \frac{1}{4}\right]$. Define $\hat{K}(\xi)=\hat{\phi}\left(2\left(\xi-\frac{1}{4}\right)\right)+$ $\hat{\phi}\left(2\left(\xi+\frac{1}{4}\right)\right)$. Then $\hat{K}(\xi)$ is supported in $\left[-\frac{1}{2}, \frac{1}{2}\right]$ and

$$
K(x)=\cos \left(\frac{\pi x}{2}\right) \phi\left(\frac{x}{2}\right) .
$$

Therefore

$$
|K(n)|= \begin{cases}\phi\left(\frac{n}{2}\right) & \text { if } n \text { is even } \\ 0 & \text { if } n \text { is odd }\end{cases}
$$

Hence,

$$
\sum_{n \in \mathbb{Z}}|K(n)|=\sum_{m \in \mathbb{Z}} \phi(m)=\hat{\phi}(0) .
$$


Now we will estimate $\|K\|_{1}$ :

$$
\begin{aligned}
\int|K(x)| d x & =\int \phi\left(\frac{x}{2}\right)\left|\cos \frac{\pi x}{2}\right| d x \\
& =2 \int \phi(x)|\cos \pi x| d x \\
& >2 \int \phi(x) \cos ^{2} \pi x d x \\
& =\int \phi(x)(1+\cos 2 \pi x) d x \\
& =\hat{\phi}(0)+\frac{1}{2} \hat{\phi}(1)+\frac{1}{2} \hat{\phi}(-1)=\hat{\phi}(0) .
\end{aligned}
$$

Thus we obtain $\int|K(x)| d x>\sum_{n \in \mathbb{Z}}|K(n)|$. This is the opposite of what we need to get. Now consider a family of kernels $K_{t}(x)=K(x+t)$ where $0 \leq t \leq 1$. We have $\left\|K_{t}\right\|_{1}=\|K\|_{1}$. On the other hand $\int_{0}^{1} \sum_{n \in \mathbb{Z}}|K(n+t)| d t=\int|K(x)| d x$. Since for $t=0$ we have $\int|K(x)| d x>\sum_{n \in \mathbb{Z}}|K(n)|$, there exists $t$ such that $\int|K(x)| d x<\sum_{n \in \mathbb{Z}}\left|K_{t}(n)\right|$. For this kernel $K_{t}$ we have $\left\|T_{\text {dis }}\right\|_{1}>\|T\|_{1}$. Basically, the idea is to show that $h(t)=\sum_{n \in \mathbb{Z}}|K(n+t)|$ is not a constant. This can also be done by calculating Fourier coefficients $\hat{h}(m)=|K|^{\wedge}(m)$ and showing that they do not vanish; take for example $K(x)=\left(\frac{\sin \frac{\pi x}{3}}{\pi x}\right)^{3}$.

Now we consider the case $p>1$. Suppose $T$ is a bounded operator from $L^{1}$ to $L^{1}$. Then $T_{d i s}$ is also a bounded operator from $\ell^{1}$ to $\ell^{1}$. As we said earlier, $\|T\|_{\infty}=\|T\|_{1}$ and $\left\|T_{d i s}\right\|_{\infty}=\left\|T_{d i s}\right\|_{1}$. Interpolating between $p=1$ and $p=\infty$, we have that $T$ and $T_{d i s}$ are bounded operators from $L^{p}$ to $L^{p}$ and $\log \|T\|_{p}$ and $\log \left\|T_{d i s}\right\|_{p}$ are convex functions. Thus $\|T\|_{p}$ and $\left\|T_{d i s}\right\|_{p}$ are continuous functions for $p \geq 1$. Let $K$ be a kernel such that $\left\|T_{\text {dis }}\right\|_{1}>\|T\|_{1}$. Then $\left\|T_{\text {dis }}\right\|_{p}>\|T\|_{p}$ for some range of $p$ : $1 \leq p<p_{0}$. We can prove this in a different way which also uses the continuous dependence of $\left\|K_{d i s}\right\|_{p}$ on $p$. Recall from (11) that $\left\|T_{d i s}\right\|_{p} \geq\left\|K_{d i s}\right\|_{p}$ and $\|T\|_{p} \leq\|K\|_{1}$. If $K$ is a kernel such that $\sum_{n \in \mathbb{Z}}|K(n)|>\int|K(x)| d x$, then

$$
\begin{aligned}
\left\|T_{d i s}\right\|_{p} & \geq\left(\sum_{n \in \mathbb{Z}}|K(n)|^{p}\right)^{\frac{1}{p}} \\
& >\int|K(x)| d x \geq\|T\|_{p}
\end{aligned}
$$

for some range of $p: 1 \leq p<p_{0}$.

Now we will do the case when the dimension $d>1$. Let $T$ be a convolution operator from $L^{p}(\mathbb{R})$ to $L^{p}(\mathbb{R})$ with kernel $K$. Define a convolution operator $\tilde{T}$ from $L^{p}\left(\mathbb{R}^{d}\right)$ to $L^{p}\left(\mathbb{R}^{d}\right)$ with kernel $\tilde{K}(x)=K\left(x_{1}\right) K\left(x_{2}\right) \cdots K\left(x_{d}\right)$. It is easy to show that $\|\tilde{T}\|_{p}=\|T\|_{p}^{d}$. The same is true for discrete analogues. Let $K$ be such a 
kernel that $\frac{\left\|T_{d i s}\right\|_{p}}{\|T\|_{p}}=B>1$. Note that $\tilde{K}_{d i s}=(\tilde{K})_{d i s}$. Then

$$
\frac{\left\|(\tilde{T})_{d i s}\right\|_{p}}{\|\tilde{T}\|_{p}}=\frac{\left\|T_{d i s}\right\|_{p}^{d}}{\|T\|_{p}^{d}}=B^{d} .
$$

It would be interesting to know if there are kernels $K$ with $\hat{K}$ supported in $\left[-\frac{1}{2}, \frac{1}{2}\right]$ such that $\left\|T_{d i s}\right\|_{p}>\|T\|_{p}$ for $1 \leq p<2$, not just for $1 \leq p<p_{0}$.

\section{REFERENCES}

[1] A. Magyar, E. M. Stein, S. Wainger, Discrete analogues in harmonic analysis: Spherical averages, Annals of Mathematics (2), 155, 2002, 189-208. MR.1888798 (2003f:42028)

E-mail address: olegk@alum.mit.edu 